\title{
Holistic Development of Mobile Applications to Support Manufacturing Processes
}

\author{
Günther Schuh, Martin Pitsch, and Michael Salmen
}

\begin{abstract}
In our everyday life smartphones and applications become more and more important. But the use of applications is not limited to private persons. Especially for the manufacturing industry supporting applications are able to enhance the productivity and to generate important advantages. This paper deals with the successful development of mobile applications to support manufacturing processes and shows the difference to regular applications. Additionally it describes a framework for developing industry applications including action fields to tackle during the development process.
\end{abstract}

Index Terms-App development, digital manufacturing, industry 4.0, Internet of Things.

\section{INTRODUCTION}

Everyday life is characterized by a constant support of smartphones as well as tablet computers and its related services. Considering western countries using a cell phone became a standard within the last few years. In 2013 more smartphones than feature phones were sold worldwide [1]. The increasing usage of smartphones and tablet computers is not only covered by the increase of devices in use, but also by the consumed data volume and the number of downloaded applications. The current data transmission is $600 \mathrm{MB}$ per month and smartphone and is predicted to increase up to 2.2 GB until 2019 [2]. Within the last years, the number of annually downloaded applications increased by at least $30 \%$ each year. Furthermore, the share of applications which are not free to download increased from $6 \%$ in 2012 to $10 \%$ in 2013. Recent surveys predict an even higher increase in the number of downloaded applications. This trend shows the importance of applications and its impact on the society [3]. An application is characterized by its intuitive usability and a high performance due to a limited range of functions. Applications invented for the consumer market are being used for a variety of purposes. The most popular categories for applications are games and education followed by entertainment and lifestyle. Applications which enhance the productivity are downloaded the least [4].

\section{A. Mobile Applications in the Industry}

Applications are highly suitable for the producing industry due to their simplicity and usability. As reported by the marketing manager of Siemens Simatic Apps, future products will be delivered together with an application supporting the user in the industry [5]. However, the holistic consideration of existing applications clearly demonstrates,

Manuscript received February 25, 2015; revised July 6, 2015.

The authors are with the Laboratory for Machine Tools and Production Engineering, Germany (e-mail: g.schuh@wzl.rwth-aachen.de). that applications have a lack of orientation towards industrial purposes. According to a trade journal for

Automation, most industrial applications are still in the phase of implementation [5]. In a survey of manufacturing companies in 2013 none of them used mobile applications in their company [6]. Compared to the market development of application the usage of mobile applications in the producing industry is nearly not existent (see Fig. 1), which is quite remarkable due to its great potential. Using an application in this context could improve the communication between employees by making coordination more efficiently, simplify the workers life by providing him with the necessary information in the right moment as well as standardize the documentation in the production process by a using a systematic tool. This is just a small extract of what mobile applications are capable of in order to enhance productivity, transparency and efficiency.

\section{Market development of applications}

Worldwide downloads

[in bn.]



Fig. 1. Market development of applications [6].

The development of an industry application is entirely different from the purpose of using applications as a private consumer. The development of industry applications requires a clear focus on the applications purpose. An industrial application is to be used for increasing the productivity as well as facilitating the everyday working life. In addition to that this application needs to fit perfectly in its company's technological and organizational environment [7].

\section{B. Action Fields}

Currently a few companies are already using smartphone and tablet applications for certain purposes. For example the German Siemens group is already offering a variety of applications for Apple products to support the use of their industrial devices. The applications are serving a variety of application fields but yet these applications do not really 
support the production process [8]. Still, there are innovative approaches to use the opportunities of applications in a more efficient way. Scientists for example developed an energy monitoring system which is able to display the energy consumption of different devices in a factory. Hereby it is possible to detect energy saving potentials [9]. Although serving similar purposes in the industry, the applications differ a lot from each other. In the current development process an application is developed only as a single application. It is not a part of a holistic system of applications which could grant immense synergy effects.

\section{REQUIREMENTS FOR THE DEVELOPMENT OF INDUSTRY APPLICATIONS}

\section{A. Characteristics of Mobile Application}

Applications are working at the interface between the human user and the device. As a tool of cooperation they can be understood as a sociotechnical system [10]. From a technical perspective the application development can be considered as a control circuit. In a control circuit the resulting output is the product of the interaction of an input variable with a regulating variable under the influence of several disturbance variables (see Fig. 2).

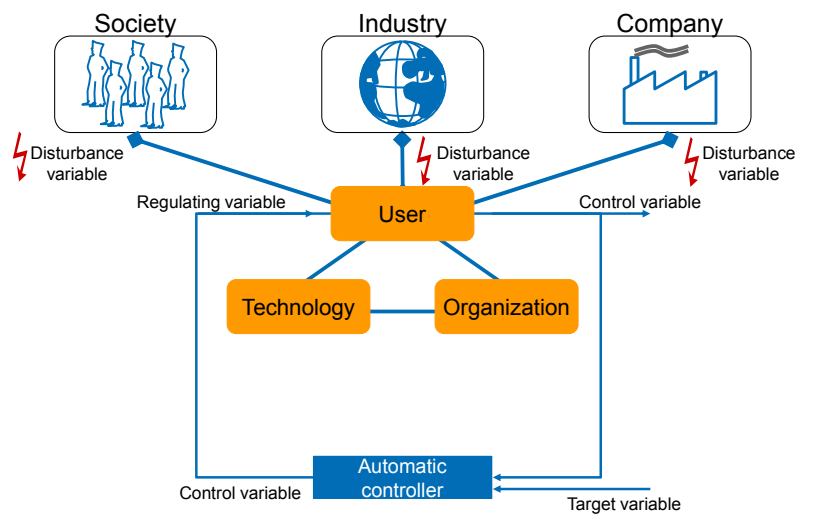

Fig. 2. Industrial application development as an output of a control circuit [11].

The main characteristics of an application can be described as its user, the applied technology and its organizational environment. The developed application can be considered as the output variable. In the context of a control circuit, the requirements to an application considering its usability can be understood as the target variable. There are three main requirements stated by society concerning the application. It has to have an easily understandable interface and also be possible to use on different devices. Furthermore it has to support and entertain the user in everyday life [12].

\section{B. Environment of Industrial Application}

In industrial purposes there are additional disturbance variables. Not only the society, but also other sources of disturbance variables have to be considered in the development of applications. In an industrial environment the specific requirements of the industry have to be considered. Furthermore there are demands which can vary between different companies. For the entire industry an enhancement of its competitiveness is essential for used applications.
Additionally the business processes have to be supported efficiently and the functionality has to be connected with current systems not operating as an isolated tool. For the individual company an important benefit component is a facilitated working process. Company specific requirements have to be grantable for individual companies. As well an important aspect is the data security of information entered in the application [13]. Currently there is no standardized approach which follows a defined requirement profile in the development of industrial applications. Hence there has to be a defined environment with requirements derived from the interaction of the framework structure.

\section{FrAMEWORK FOR THE HOLISTIC DEVELOPMENT OF INDUSTRY APPLICATIONS}

As mentioned before the low number of existing industry applications is a result of the missing systematic approach for developing industry applications. It is both necessary to consider the characteristics of regular applications and the characteristics of the industry. A successful approach for a holistic development of industry applications illustrates the framework shown below (see Fig. 3). The framework integrates the two main groups of challenges mentioned in chapter 2: The application structure with its dimensions Technology, Organization, and User is the framework's basis.

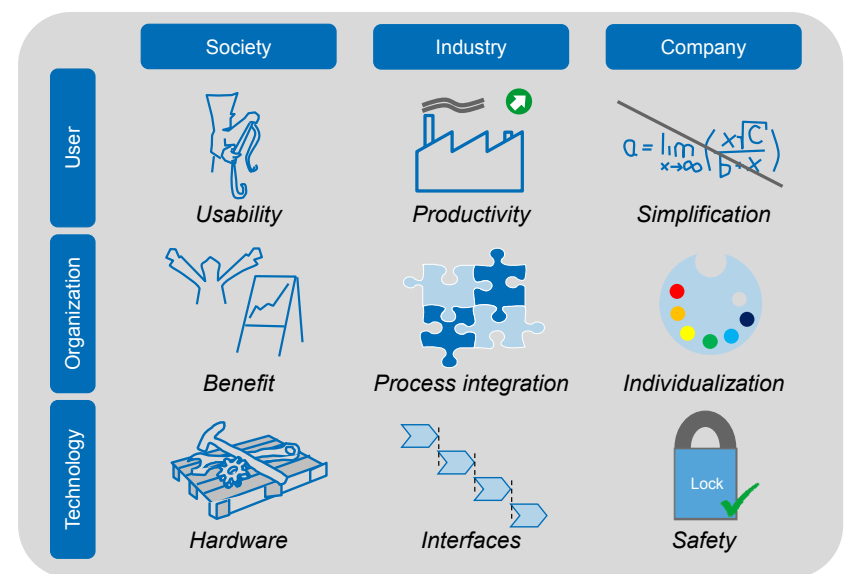

Fig. 3. Framework for the holistic development of industry applications [11].

The integration of the disturbance variables Society, Industry, and Company leads to a matrix with nine fields of action. Every single field of action needs to be considered and tackled during the entire development process. The following part will describe the three fields of actions within the variables Society, Industry, and Company.

Variable 1: Society. Combining the variable society with the dimensions of the application structure leads to three fields of action: Usability, Benefit, and Hardware.

Usability: In terms of handling an industry application needs to imply three main characteristics: At first a self-explanatory setting is necessary to not discourage the user. An intuitive handling using symbols or gestures simplifies the usability and therefore lowers the entry barriers for potential users. Furthermore the industry application with its features and settings needs to be adjusted to the specific target group. Their characteristics as well as their needs have 
to be considered during the entire development process.

Benefit: The industry application should focus on a certain application context, for example process monitoring or failure analysis. Offering multiple functions in different contexts leads to complications. In conjunction with the focus on a certain application context the specific benefit of the industry application needs to be clear. A lack of clarity about the applications benefit will decrease the willingness of its usage. As the information and therefore the specific benefit needs to be delivered to the user, a suitable graphic user interface is important. It should either be informative, stylish or intuitive, depending on the applications benefit.

Hardware: The industry application needs to fit the devices the target group is using. An analysis of the devices in terms of operation system and standard equipment is inevitable. The market development as well as future trends could be essential for the success of an industry application and should therefore be considered as well. Furthermore, the modularization and expandability of the devices' hardware should be taken into account, for example the possibility of using USB drives, SD cards or scanners.

Variable 2: Industry. Three fields of action exist concerning the integration of the variable industry into the three dimensions User, Technology, and Organization of the application structure: Productivity, Process integration and Interfaces.

Productivity: The productivity as a field of action implies two main characteristics: At first the industry application needs to gain productivity. Examples are the increase of employee efficiency using the application on electronic devices or the improvement of information flows due to real time reports. Furthermore a well-defined target of the productivity increase has to be set. It is necessary to measure the success or failure of the application in use.

Process integration: The integration into the industry's processes as well as the holistic understanding of its business is important for the industry application. Different branches require different solutions, and even the companies and their businesses within a certain branch can differ in multiple characteristics. Interdisciplinary development teams are therefore necessary to implement the process integration through interaction with the industry application.

Interfaces: The consideration of different types of interfaces enables the application to connect with the existing systems. It is necessary to implement standard interfaces in the particular industry in order to avoid isolated solutions. It is furthermore necessary to define the interfaces in the context of the industry application. The existing types of interfaces, such as hardware interfaces, software interfaces, network interfaces and user interfaces need to be evaluated with regard to use case of the application.

Variable 3: Company. Simplification, Individualization, and Safety are the fields of action to tackle considering the dimension company with the three dimensions of the application structure.

Simplification: Target of the simplification is to simplify the work using numerous methods and approaches. Possible solutions are e.g. data mining to filter important information from data sets, digitalization to ease inputs and outputs or gamification to increase the employee's motivation by using various symbols, community collaboration and feedback. Various solutions should be considered during the development process of industry applications.

Individualization: Three main characteristics derive from the individualization: At first the industry application needs to obtain a corporate design. The individual adjustment of colors and logos makes the industry application unique and at the same time meets the company's requirements in terms of corporate design and style. Furthermore, the flexibility of front- and backend enables a flexible design of the graphic user interface. A modular platform as the third characteristic ensures the expandability of functions and therefore lowers the barriers for the development as well as the improvement of the industry applications.

Safety: As the industry application is using highly confidential company information, it is necessary to take several precautions in the development phase: Referring to the Secure Development Lifecycle (SDL) developers, software architect and security experts need to analyze specific threats, define actions for each threat and take these threats into account during the implementation phase. The main target is to develop an application which is safe against unauthorized access from both inside and especially outside the company.

\section{SUMMARY AND OUTLOOK}

The general aim was to provide a guideline for the successful development of industry applications. Therefore, the differences between regular applications and industry applications were outlined. Thus, a framework for developing industry applications including action fields to tackle during the development process was generated and explained. The presented approach is a basis for a systematic development of applications used in the producing industry. Nevertheless, several fields of action need to be addressed to ensure the role of the producing industry sector. The development process for industrial applications will undergo further development phases. A new word generated for the future application development is open-core engineering [14]. Here, the basic function of an application stays the same, while companies can adjust aspects to have their individual requirements fulfilled. The presented framework for the development of industrial applications remains untouched as the focus of core-engineering will be general tools and systems. The distribution of industrial applications is considered as another future challenge [15]. The existing industrial applications are already being sold in the application stores of Apple and Google. Developing this sales model to a specific application store only for industrial applications could increase the competition and accelerate the progress in the development of industrial applications. Fields of actions, which are more specific to a certain context are the digitalization of ordering parts and the improved integration of individualization. Another possible aspect for industrial applications is to only have one controlling device running an application which is then in charge of controlling different manufacturing units. Specific applications can soon be considered as a tool fostering client relationships [16]. The technology-driven companies need to participate in the 
trends mentioned above in order to obtain a pioneer position in the future. The use of industrial applications during the product's lifecycle helps to improve the process in a technical and economical way. The change to an application supported manufacturing process ensures the future competitiveness.

\section{REFERENCES}

[1] Gartner, "Market share analysis: Mobile phones worldwide, 4Q13 and 2013," 2014.

[2] Ericsson, "Ericsson mobility report, study of ericsson on the smartphone market," November 2013, p. 11.

[3] Gartner, "Forecast: Mobile app stores, worldwide, 2013 update," 2013.

[4] 148apps.biz. (2014). App store metrics, evaluation of applications downloaded in the iTunes app store. [Online]. Available: http://148apps.biz/app-store-metrics/?mpage=catcount

[5] H. GmbH, "IEE app-guide 2013," German Professional Magazine on Applications in Machine and Plant Engineering, HüthigVerlag, Heidelberg, July 2013, pp. 4-7.

[6] WBA Werkzeugbauakademie, "Knowledge transfer in the tool manufacturing industry," Aachen, 2013.

[7] R. Stair and G. Reynolds, "Principles of information systems," Cengage Learning, 2014, p. 85.

[8] Siemens. Overview on industrial applications. [Online]. Available: http://w3.siemens.com/topics/global/en/apps/industry/Pages/default.as $\mathrm{px}$

[9] c4c engineering. (2013). Intelligente Apps fürMittelstand und Industrie. [Online].

Available:

http://www.c4cengineering.de/aktuell/intelligente-apps-fuer-mittelsta nd- und-industrie.html

[10] E. Trist and K. Bamforth, "Some social and psychological consequences of the long wall method of coal getting," 1951, pp. 3-38.

[11] M. Pitsch, "Holistic industry applications," Aachen, 2014.

[12] C. Banga and J. Weinhold, "Essential mobile interaction design: Perfecting interface design in mobile design," Pearson Education, 2014, p. 65.

[13] ISC2, "The ten best ways for secure mobile development," 2012.

[14] Bosch Rexroth. (February 5, 2015). [Online]. Available: http://www.boschrexroth.com/en/xc/products/engineering/opencoreen gineering/the-features-of-open-core-engineering/the-features-of-opencore-engineering

[15] P. Sharpe: "The growth of mobile \& tablet applications within the financial industry," Study of Morgan Stanley, 2012, p. 11.
[16] P. Sharpe, "The growth of mobile \& tablet applications within the financial industry," Study of Morgan Stanley, 2012, p. 8.

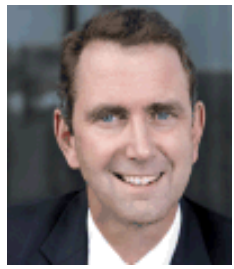

Günther Schuh studied mechanical engineering and economics at the RWTH Aachen from 1978 until 1985. He received his doctorate in 1988 following his research fellowship at the WZL, where he was also the head engineer until 1990, under Professor Eversheim.

$\mathrm{He}$ lectured manufacturing economics and industrial management at the University of St. Gallen (HSG) from 1990 until becoming a professor for economic production management and the co-director of the Institute for Technology Management in 1993. He also founded the GPS Group in Wuerselen, St. Gallen and Atlanta.

Professor Schuh was awarded the Otto-Kienzle Memorial Coin by the Scientific Society for Production Technology in 1991. He has often been commended for his scientific work at the Swiss Technology Competition. His most important research work includes various methods and instruments related to complexity management, resource-oriented process cost calculations, participative change management and the virtual factory concept. Professor Schuh is also a member of several boards of directors and councils and was the deputy headmaster for industry and economics of the RWTH Aachen from 2008 to 2012.

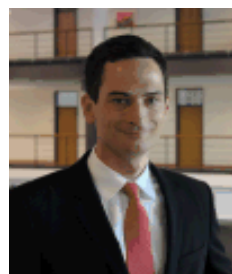

Martin Pitsch studied mechanical engineering and economics at the RWTH Aachen and at the Chalmers University of Technology Göteborg. He received his doctorate in 2014 following his research fellowship at the WZL, where he currently is employed as the head engineer and the head of the Department for Business Development. Dr. Pitsch is specialized in the development of organizations, strategic management and value stream oriented production planning and steering.

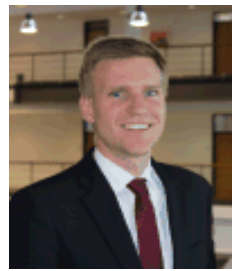

Michael Salmen studied mechanical engineering and economics at the RWTH Aachen. He currently works as a research fellow at the WZL Aachen in the Department for Business Development as a group leader. Mr. Salmen is specialized in production networks, benchmarking and digital production planning and steering. 